\title{
3-D structural vibration measurement using digital photogrammetry
}

\author{
YANG Zhao \& WANG Cong \& GAO Jingbo
}

\author{
School of Astronautics, Harbin Institute of Technology, Harbin 150001, China
}

Keywords: Digital photogrammetry; Vibration measurement; Dynamic response; Imaging techniques

\begin{abstract}
In this paper, a methodology has been developed to measure the dynamic vibrational displacements of a structure by using digital photogrammetry. Sequential stereo images of the vibrating structure with target mark can be obtained by two industrial cameras. According to the binocular vision theory, the spatial coordinates of the target point can be computed, then the displacement of a structure in 3-D space, such as, the planar and in-depth displacements, can be recorded. So the 3-D dynamic motion of a structure is obtained. This method doesn't need to attach sensors to the structure which may cause negative effects to the structure's motion. Even in some special situations, i.e., in space or other extreme situations, which are not convenient to use accessible facilities, the proposed method is almost unaffected. To validate the proposed method, a experiment is performed.
\end{abstract}

\section{Introduction}

When measure the vibration of a structure, there a two methods are generally used. One is the contact type which uses accelerometers or displacement sensors. These facilities need to be attached to the structure so that the dynamic characteristics may have changes. Moreover, in many cases, this method is not desirable when access to the structure is prohibited or dangerous. For the non-contact type, laser displacement sensor usually is used. However, it is relatively expensive and unsatisfactory when the vibration is large. What's more, only a single direction of vibration of a specific point on a structure usually can be obtained by both methods.

Digital photogrammetry is a measurement technique with many advantages compared to the methods described earlier when measure the vibration of a structure. Photogrammetry uses photographs to measure a 3-D object by using its 2-D images. A spatial coordinate time history of the object can be obtained by using sequences of images. According to the types of photographs used in analysis, the photogrammetric technique can be defined as aerial or terrestrial. If the images which are obtained from aircrafts, satellites, or other very high place, the photogrammetric technique is aerial. On the other hand, if the images which are obtained from ground-based cameras, the photogrammetric technique is terrestrial.

Photogrammetry has been used to measure the dynamic displacement of structures. Olaszek investigated the dynamic characteristics of bridges using the method which incorporated the photogrammetric principle with the computer vision technique. Patsias and Staszewski used photogrammetry to analyze the mode shapes of a cantilever beam. Ozbek and Rixen obtained the dynamic behavior of wind turbines by photogrammetry. Xu et al. developed a method to give the modal analysis of large flexible structure using photogrammetric technique. In these paper, the authors all chose the commercial high-speed camera, and they didn't give a very detailed explanation about how they use the theory of photogrammetry to measure the vibration.

In this study, a photogrammetric method is developed for measuring 3-D vibration response of a structure. Two same industrial cameras are chosen to acquire images, and each one has a maximum shutter speed of 200 frames per second(fps). But, in this study, each camera only takes 30 fps when it acquires images. This shutter speed can be taken by many low-level cameras which can be easily bought by general users. To calibrate the cameras, ZHANG Zhengyou's calibration method is utilized. This method is easily implemented by using a planar pattern which is shown at some different orientations. Two cameras are triggered by a signal generator, then they begin to acquire 
images synchronously. The 3-D vibrational displacements of the target are obtained using these picture. Finally, to validate the proposed method, a laboratory test for a vibrating cantilever beam is performed.

\section{Photogrammetric measurement system}

The proposed photogrammetric method is also called a photogrammetric measurement system which consists of two industrial cameras, a calibration plate, and a few mark points which are attached on test structure. The key steps of this method are camera calibration, finding target point, and 3-D point reconstruction.

Camera calibration. Camera calibration is utilized to determine the intrinsic parameters and extrinsic parameters. Intrinsic parameters include the focal length, the principal point, and the lens distortion. Extrinsic parameters include translational coordinates and three-axes rotational angles which are the rotation and translation which relates the world coordinate system to the camera coordinate system. According ZHANG Zhengyou's calibration theory, the relationship of the vector of 2-D point in the image coordinate which is defined by pixels can be expressed as:

$$
Z_{c}\left[\begin{array}{c}
u \\
v \\
1
\end{array}\right]=\left[\begin{array}{cccc}
f / d x & s & u_{0} & 0 \\
0 & f / d y & v_{0} & 0 \\
0 & 0 & 1 & 0
\end{array}\right]\left[\begin{array}{cc}
\boldsymbol{R} & \boldsymbol{t} \\
\boldsymbol{0}^{T} & 1
\end{array}\right]\left[\begin{array}{c}
X_{w} \\
Y_{w} \\
Z_{w} \\
1
\end{array}\right]
$$

where $\mathrm{u}$ and $\mathrm{v}$ are the pixel coordinates in the image coordinate system; $X_{w}, Y_{w}, Z_{w}$ are the coordinates in the world coordinates system; $Z_{c}$ is the scale factor; $\mathrm{f}$ is the focal length; $\mathrm{dx}$ and dy are the distance between the two adjacent pixels; $u_{0}, v_{0}$ are the coordinates of the principal point in terms of pixel dimensions; $\mathrm{R}$ is the rotation matrix; and $\mathrm{t}$ is the translation matrix.

The calibration plate is shown as Fig. 1. To calibrate the camera, at least 8 images of different orientations are needed to calculate the intrinsic parameters and extrinsic parameters.

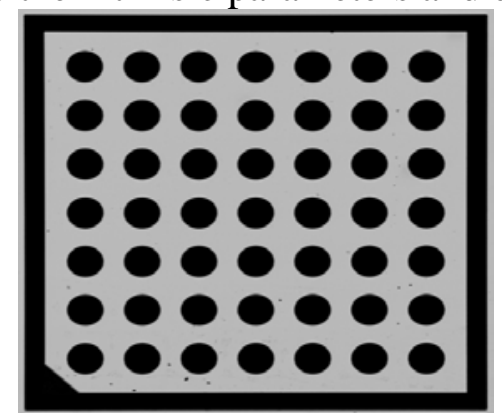

Fig. 1 Calibration plate

Finding target point. In this study, the solid circles is utilized as target points shown as Fig.2. First, the circles are found by using the theory of edge detection. Then the coordinates of centers can be obtained(Fig.3) so that these coordinates can be used to process the 3-D point reconstruction.

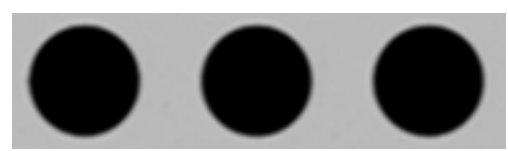

Fig. 2 Target points

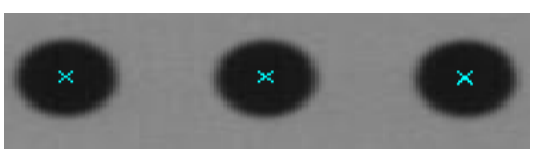

Fig. 3 Centers found by computer

Reconstruction of 3-D point. Equation (1) can be written as: 


$$
Z_{c}\left[\begin{array}{c}
u \\
v \\
1
\end{array}\right]=\boldsymbol{N M}\left[\begin{array}{c}
X_{w} \\
Y_{w} \\
Z_{w} \\
1
\end{array}\right]=\boldsymbol{Q}\left[\begin{array}{c}
X_{w} \\
Y_{w} \\
Z_{w} \\
1
\end{array}\right]
$$

where $\mathrm{N}$ is the matrix of intrinsic parameters; $\mathrm{M}$ is the matrix of extrinsic parameters; and $\mathrm{Q}$ is the matrix of projection matrix. Because there are two cameras acquiring images synchronously, a equation system can be obtained as:

$$
\left\{\begin{array}{c}
Z_{c 1}\left[\begin{array}{c}
u_{1} \\
v_{1} \\
1
\end{array}\right]=\boldsymbol{N}_{1} \boldsymbol{M}_{1}\left[\begin{array}{c}
X_{w} \\
Y_{w} \\
Z_{w} \\
1
\end{array}\right]=\boldsymbol{Q}_{1}\left[\begin{array}{c}
X_{w} \\
Y_{w} \\
Z_{w} \\
1
\end{array}\right] \\
Z_{c 2}\left[\begin{array}{c}
u_{2} \\
v_{2} \\
1
\end{array}\right]=\boldsymbol{N}_{2} \boldsymbol{M}_{2}\left[\begin{array}{c}
X_{w} \\
Y_{w} \\
Z_{w} \\
1
\end{array}\right]=\boldsymbol{Q}_{2}\left[\begin{array}{c}
X_{w} \\
Y_{w} \\
Z_{w} \\
1
\end{array}\right]
\end{array}\right.
$$

$\boldsymbol{Q}_{1}$ and $\boldsymbol{Q}_{2}$ can be computed by camera calibration, and $Z_{\mathrm{cl}}, Z_{\mathrm{c} 2}$ can be eliminated. And then, there are 3 unknown numbers and 4 equations. The world coordinates are obtain by solving the equation system using least squares solution. By using the image sequences of the two cameras, the 3-D displacement curves can be acquired.

\section{Experiment}

To validate the proposed method, a experiment for a vibrating cantilever beam is performed. In this experiment, two industrial cameras, AVT Manta G-419, which can take $30 \mathrm{fps}$,are used for image sequence acquisition. The resolution is 1200 pixels $\times 800$ pixels. The target points were placed on the cantilever beam as shown in Fig. 4. The base line of two cameras were placed at about $1.8 \mathrm{~m}$ away from the target and $0.45 \mathrm{~m}$ from each other(see Fig.5).

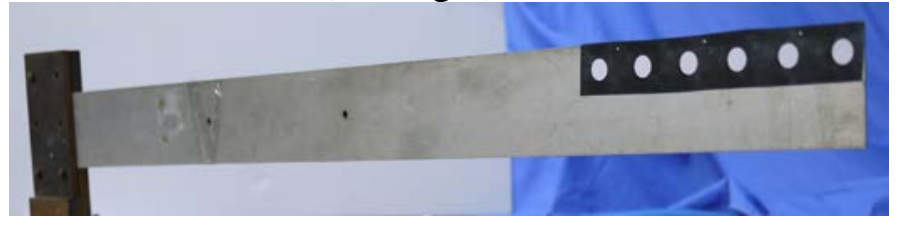

Fig. 4 The position of the target

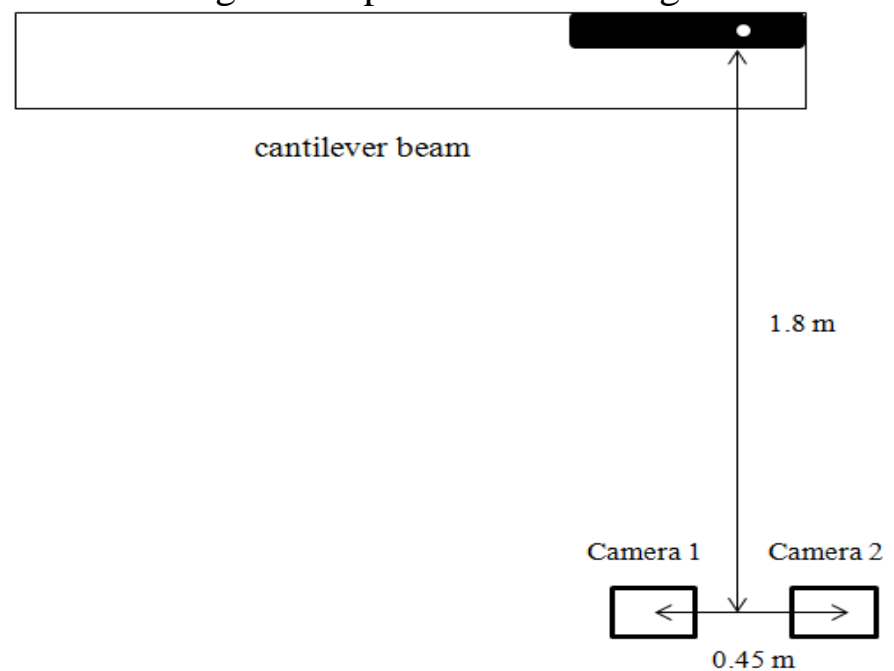

Fig. 5 The position of the two cameras

To calibrate the cameras, 9 images of calibration plate which was placed in different orientations(see Fig.6). 


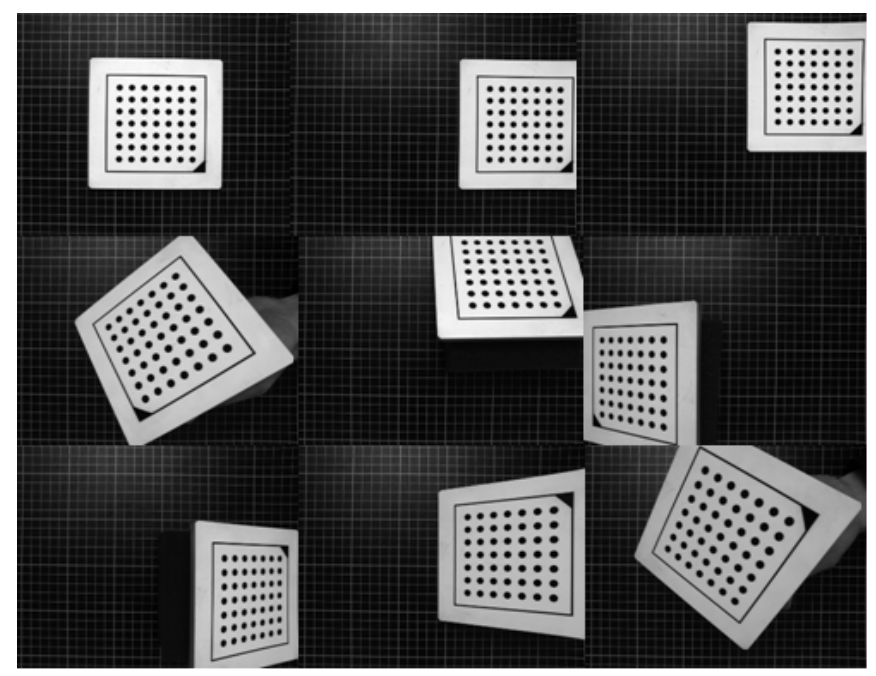

Fig. 6 Positions of calibration plate

According to the ZHANG Zhengyou's theory, the intrinsic parameters and extrinsic parameters of the two cameras in Table 1 were determined by using the images of the calibration plate.

Table 1 Intrinsic parameters and extrinsic parameters of the two cameras

\begin{tabular}{|c|c|c|c|c|c|c|c|c|c|c|}
\hline & $\begin{array}{c}\mathrm{f} \\
(\mathrm{mm})\end{array}$ & $\begin{array}{c}\mathrm{dx} \\
\text { (um) }\end{array}$ & $\begin{array}{c}\text { dy } \\
\text { (um) }\end{array}$ & $\begin{array}{c}\mathrm{s} \\
(\mathrm{mm}) \\
\end{array}$ & $\begin{array}{c}u_{0} \\
(\mathrm{~mm})\end{array}$ & $\begin{array}{c}v_{0} \\
(\mathrm{~mm})\end{array}$ & \multicolumn{3}{|c|}{$\mathrm{R}$} & $\begin{array}{c}\mathrm{t} \\
(\mathrm{mm})\end{array}$ \\
\hline \multirow{2}{*}{$\begin{array}{l}\text { Left } \\
\text { camera }\end{array}$} & 12.7 & 5.48 & 5.5 & 0 & 297.8 & 230.1 & 0.989 & 0.238 & -0.154 & 448 \\
\hline & & & & & & & -0.027 & 0.999 & -0.020 & -2.84 \\
\hline $\begin{array}{l}\text { Right } \\
\text { camera }\end{array}$ & 13.3 & 5.47 & 5.5 & 0 & 306.3 & 224.0 & 0.153 & 0.024 & 0.988 & -8.93 \\
\hline
\end{tabular}

Then, the two cameras began to acquire images synchronously, and the images of the two cameras at one same moment were shown in Fig.7 and Fig.8.

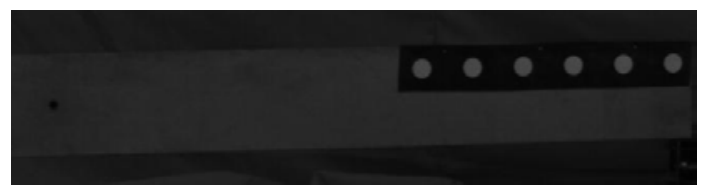

Fig. 7 Images of left camera at one moment

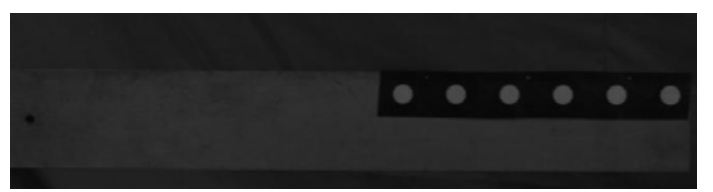

Fig. 8 Images of right camera at one moment 


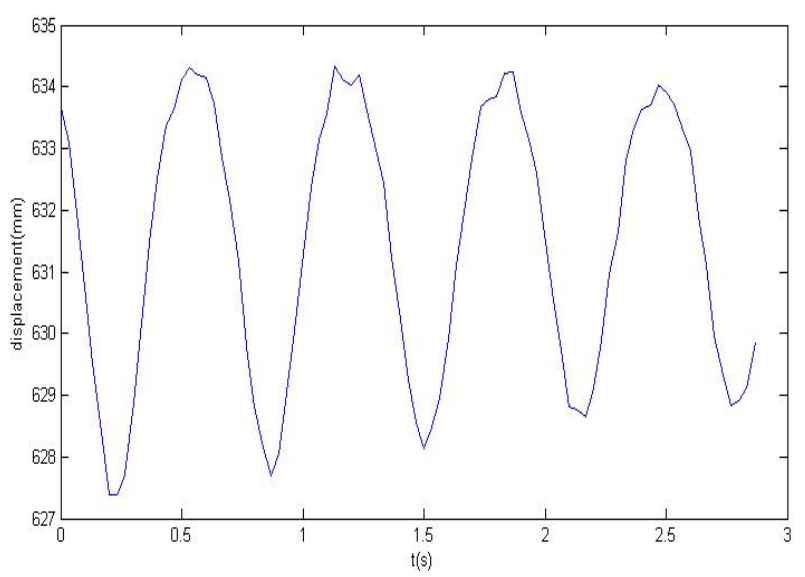

Fig. 9 (a) X-displacement of the rightmost target point

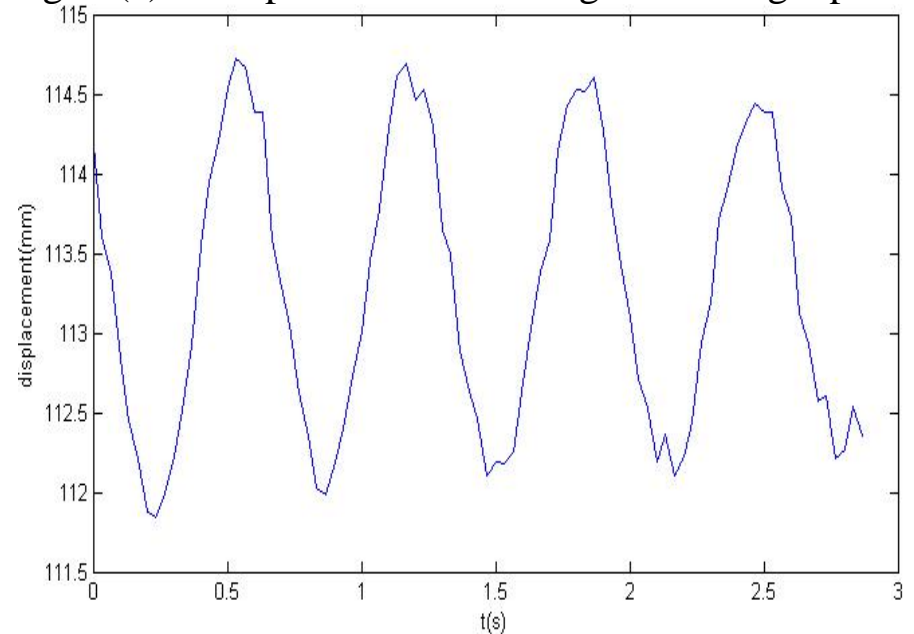

Fig. 9 (b) Y-displacement of the rightmost target point

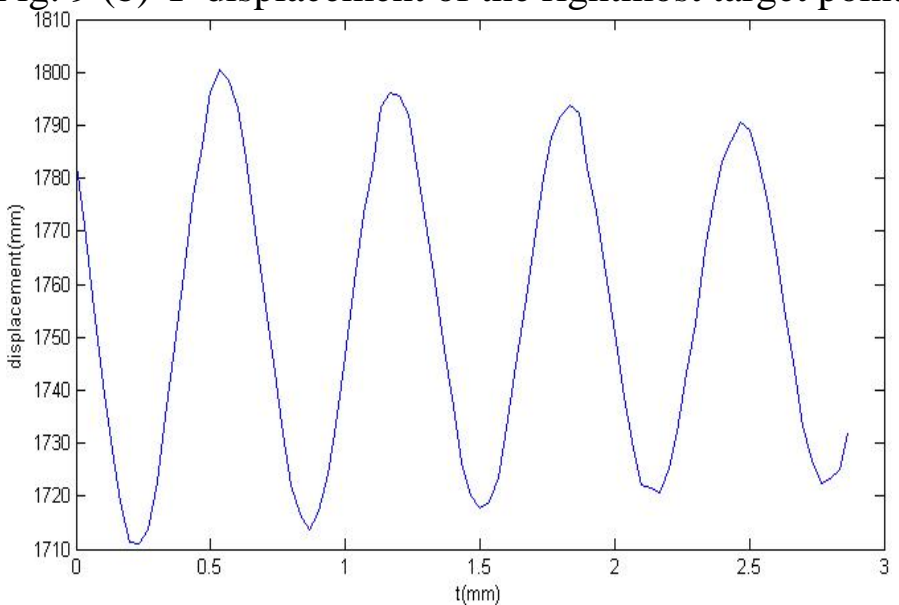

Fig. 9 (c) Z-displacement of the rightmost target point

Fig. 9 showed the 3-Ddisplacements (X,Y and $\mathrm{Z}$ ), at the rightmost target point.

If more targets point are attached to the cantilever beam, we can easily obtain the 3-D dynamic deformation shape of the cantilever beam by using the 3-D displacement data.

\section{Conclusion}

In this paper, a dual-camera photogrammetric method for 3-D vibrational displacement was presented. The proposed method doesn't use a very high shutter speed, but use shutter speed of 30 fps. The proposed method is based on the theories of photogrammetry and computer vision, and it includes the following steps: camera calibration, finding target point, and 3-D reconstruction of target points. An experiment was performed to measure the 3-D vibration of a cantilever beam. 
Comparing with the traditional methods, this photogrammetry method has many advantages because it doesn't need to contact with the structure and is easy to be performed. This method will have a very wide range of industrial areas. For instance, it can be used in monitoring the antenna on a satellite and the other large-scale flexibility structures.

\section{References}

[1] LIU Yan \& LI Tengfei 2014. Research of the improvement of Zhang's camera calibration method. Optical Technique 40(6): 565-570.

[2] Olaszek P. 1999. Investigation of the dynamic characteristic of bridge structures using a computer vision. Measurement 25(3): 227-236.

[3] Ozbek M. \& D. J. Rixen D. J. \& Erne O. \& Sanow G. 2010. Feasibility of monitoring large wind turbines using photogrammetry. Energy 35: 4802-4811

[4] Patsias S. \& Staszewski W. J. 2002. Damage detection using optical measurements and wavelets. Struct. Health Monit 1(1): 7-22.

[5] XU Chang \& WANG Cong \& GAO Jingbo \& ZHANG Chunfang 2014. Videogrammetric based modal identification of on-orbit flexible structures. Journal of Harbin Institute of Technology 46(11): 17-23.

[6] XIAO Chuanmin \& SHI Zelin \& XIA Renbo \& WU Wei 2014. Edge-detection algorithm based on visual saliency. Information and Control 43(1): 9-13. 